\title{
Posibilidades de implantación de una plataforma virtual para la mejora de la comercialización de los cítricos en Andalucía. Necesidades de información de los productores y exportadores
}

\author{
M.D. García-Brenes ${ }^{1, *}$, P. Rallo², F.J. Arenas-Arenas², A. Morales-Sillero² \\ y M.P. Suárez ${ }^{2}$ \\ 1 Departamento de Economía Aplicada II. Escuela Técnica Superior de Ingeniería Agronómica (ETSIA). \\ Universidad de Sevilla. Carretera de Utrera Km 1. 41013. Sevilla \\ 2 Departamento de Ciencias Agroforestales. Escuela Técnica Superior de Ingeniería Agronómica (ETSIA). \\ Universidad de Sevilla. Carretera de Utrera Km 1. 41013. Sevilla
}

\section{Resumen}

Andalucía es la segunda región productora de cítricos de España, teniendo por tanto una extraordinaria importancia económica y social. Sin embargo, la cadena de valor de los cítricos comprende un sector productor bastante atomizado frente a unos compradores muy concentrados y organizados, y un mercado poco transparente. El objetivo de esta investigación fue determinar las necesidades de información de los principales agentes implicados en la cadena de valor de los cítricos y estudiar las posibilidades que tendría la implantación de una plataforma virtual para mejorar las decisiones empresariales en la comercialización de los productos. Para ello, se emplearon fuentes primarias, centradas en la realización de entrevistas a expertos de la producción de cítricos en Andalucía, segmentando el análisis sobre la base de la naturaleza jurídica y la actividad de los entrevistados. Esta acción estratégica puede ser viable puesto que hay una clara demanda de información por parte de la población encuestada, que representa el $65 \%$ de la producción citrícola andaluza. Además, se constató que el modelo de gestión debería ser mixto, públicoprivada. La responsabilidad de la misma debería ser privada, ya que en general se percibió cierta reticencia en la Administración. La mayoría de los encuestados estarían dispuestos a pagar por los servicios que suministre la plataforma, si bien señalaron restricciones en cuanto al acceso, calidad del servicio y precio.

Palabras clave: Cadena de valor, distribución, formación de precios, cooperativas, servicios.

\begin{abstract}
Possible implementation of a virtual platform to improve citrus marketing in Andalusia (southern Spain). Information needs of producers and exporters

Andalusia is the second largest citrus producer in Spain, and thus, the citrus industry has an extraordinary economic and social importance in the region. However, the citrus value chain comprises a scattered primary sector in contrast to a very concentrated and well organized purchase and distribution sector, as well as a non-transparent market. The aim of this research was to explore the information needs of the different agents in the citrus value chain and to study the possibilities of implementing a
\end{abstract}

* Autor para correspondencia: mdgarcia@us.es http://dx.doi.org/10.12706/itea.2015.018 
virtual platform to share information in order to improve their marketing decisions. Primary sources were used for this work: a survey was conducted among citrus production experts in Andalusia. Survey results were analysed according to the legal nature and the activity of the respondents. The strategic action of implementing an information platform may be feasible since a clear demand for information has been detected from the survey population which represents $65 \%$ of the Andalusia citrus production. In addition, a public-private management model was preferred by the respondents to control the platform although the responsibility for it should be private. The majority of respondents would be willing to pay for the services provided by the platform, although some aspects regarding access restrictions service quality and price should be considered.

Key words: Value chain, distribution, price formation, cooperatives, services.

\section{Introducción}

España es el sexto productor y el primer exportador mundial de naranjas y mandarinas en fresco (FAO, 2013). Andalucía se ha posicionado en los últimos años como la segunda región productora de cítricos en España, detrás de la Comunidad Valenciana. Además, este protagonismo se consolidará en los próximos años en relación al resto de zonas productoras españolas (Arenas et al. 2009, 2011; Arenas y Hervalejo, 2013). Este aumento está relacionado con el incremento de la superficie cultivada que se ha extendido de unas 47.000 ha en el año 2.000 a 86.162 ha en 2010 (Consejería de Agricultura Pesca y Desarrollo Rural, 2013). A nivel provincial destaca Sevilla con 29.699 ha y Huelva con 20.152 ha, le siguen Málaga, Córdoba y Almería con un total de 32.068 ha (MAGRAMA, 2013).

Este sector alcanzó en el 2011 un valor de la producción de 681 millones de euros, lo que supone el $7 \%$ de toda la Producción de la Rama Agraria Andaluza, y generó 3,9 millones de jornales/año, ocupando el tercer puesto como sector agrario generador de empleo (Consejería de Agricultura, Pesca y Desarrollo Rural, 2013).

En relación al destino de la producción citrícola andaluza destaca el consumo interior en fresco $(50,7 \%)$, seguido de la industria de transformación $(24,6 \%)$ y la exportación
$(19,5 \%)$. Aunque gran parte del consumo interior acaba en la exportación a través de empresas localizadas en otras comunidades autónomas. La Unión Europea (UE) es el principal cliente y representa el $95 \%$ del total de las exportaciones. No obstante, cada vez es mayor la competencia de otros países productores del Mediterráneo, debido a la formalización de acuerdos con terceros países que favorecen la entrada de sus producciones en los mercados de la UE (Eraso, 2010).

La cadena agroalimentaria de los cítricos es bastante compleja pues se obtienen productos en fresco (naranjas, limones, mandarinas, etc.) y productos transformados (fundamentalmente zumos). Además, en el proceso de producción, transformación y comercialización participan productores, organizaciones de tipo cooperativo, exportadores y la Distribución Moderna (supermercados e hipermercados) que están interrelacionados pero tienen diferentes intereses. Desde esta perspectiva, el conocimiento de una serie de variables, como los precios en origen, precios en destino, normativa, costes, etc., resulta básico para la rentabilidad de los operadores, y principalmente para los productores agrarios, pues constituyen el eslabón más débil de la cadena agroalimentaria de los cítricos frente a la fuerte concentración de la Distribución Moderna, lo que implica grandes desequilibrios en sus relaciones. Eraso (2010) señala que los principales problemas de los productores son: la opa- 
cidad del mercado, los elevados costes de producción y de recolección, las altas garantías de cobro de algunas operaciones y los altos estándares de calidad exigidos por la comercialización y los consumidores.

Asimismo, los cambios acaecidos en el pasado más reciente han configurado un sistema agroalimentario en el que la Distribución Moderna domina y dirige la cadena agroalimentaria. Tanto es así que, en 2009, la Distribución Moderna alcanzó el $63,1 \%$ de las ventas en alimentación a los hogares españoles, mientras que las tiendas tradicionales tuvieron una cuota del $27,7 \%$ y otros canales de comercialización el 9,2\% restante (Ministerio de Industria, Turismo y Comercio, 2010). Sin embargo, las peculiaridades de la comercialización de la alimentación fresca y el importante papel de la red pública de Mercas que abastecen al pequeño comercio hacen que las tiendas tradicionales tengan un importante peso específico en la venta de la alimentación fresca, y que el canal tradicional conserve su protagonismo frente a los formatos de la Distribución Moderna. Así, las tiendas tradicionales tuvieron una cuota de mercado del $45,5 \%$ en la alimentación en fresco en 2009, y la Distribución Moderna del 43\% (Ministerio de Industria, Turismo y Comercio, 2010).

Las ventas de frutas y hortalizas constituyen un sector estratégico para la Distribución Moderna puesto que son unos alimentos muy importantes en la dieta alimenticia para los hogares españoles y acuden frecuentemente a los establecimientos comerciales para comprarlos. Son productos clave ya que el $64 \%$ de los españoles compran frutas y verduras entre dos y tres veces por semana. España es el país de Europa que más valora el consumo de productos frescos. Si las cadenas de la Distribución Moderna logran atraer al cliente para que compren estos productos, se aseguran dos visitas a la semana. $Y$ con este reclamo, los clientes suelen comprar otros productos en el establecimiento (Delgado, 2013).
Una de las consecuencias más notables de la concentración de la distribución comercial es el incremento de su poder de negociación frente a los proveedores y suministradores. La existencia de una oferta muy atomizada y una demanda muy concentrada en el mercado hace que acabe dándose de facto un oligopolio de demanda que genera un evidente desequilibrio en la cadena de suministro. Las consecuencias más destacadas de esta situación es la imposición de protocolos muy exigentes (condiciones de entrega, estándares de calidad y seguridad, práctica de pago, etc.) a sus proveedores, y una presión a la baja de los precios pagados (Aznar-Sánchez et al., 2013).

El análisis de la evolución de la parte del precio pagado por los consumidores que revierte en los productores de productos frescos, revela que entre 2004 y 2011, la participación media de los productores en los precios finales fue del $33 \%$. En el caso de los cítricos, sólo alcanzó el 14,3\% (Rebollo-Arévalo, 2012). Existe por tanto, una importante fuga de valor añadido que se escapa de los productores de productos frescos y que llega a otras etapas de la cadena agroalimentaria.

En el entorno actual de creciente competitividad en el que se encuentra el sistema agroalimentario andaluz y en particular la cadena de valor de los cítricos, las diferentes fases se coordinan para responder de un modo eficiente a las necesidades de los consumidores. Las fases de la cadena agroalimentaria se integran mediante relaciones de capital, mecanismos contractuales $u$ otras formas de integración vertical, lo cual requiere compartir información y métodos de gestión. En todos estos procesos siempre hay una fase que lidera, alejándose cada vez más de las fases más próximas a la producción agraria y afianzándose en la Distribución Moderna, que finalmente es la que mantiene las relaciones con el consumidor y debe captar sus preferencias y cambios de gustos (Langreo, 2012). Al mismo tiempo, la formación de estas ca- 
denas globales supone una profunda segmentación del mercado, formando básicamente dos grupos operadores: uno muy reducido con altos niveles de rentabilidad e información y otro muy numeroso, marginado y con grandes problemas para continuar con su actividad.

A pesar de que en los últimos años la Administración nacional y autonómica han desarrollado diversos mecanismos para favorecer la transparencia del mercado y la difusión de los precios en origen y en destino, un número importante de citricultores venden a resultas, es decir entregan la producción sin saber el precio al que le van a liquidar la misma.

En este contexto, el objetivo de este trabajo ha sido identificar las necesidades de información y tecnología de los componentes de la cadena de valor (fundamentalmente productores y exportadores) del sector de los cítricos en Andalucía, y considerar la posible implantación de una plataforma de servicios para responder a sus demandas utilizando el marco teórico del concepto de la cadena de valor y la teoría de las decisiones empresariales.

\section{Material y métodos}

Sobre el concepto de cadena agroalimentaria

La producción de alimentos se ha convertido en un proceso complejo. En la actualidad, la agricultura suministra las materias primas a la industria agroalimentaria que las transforma en productos finales, y adquiere unos inputs intermedios que son fundamentales para el proceso agrario. Esto ha provocado la progresiva integración del sector agrario en otros sectores de la economía así como una menor participación de la agricultura en el valor final de los alimentos (Mili, 1996).
En este contexto es necesario introducir el concepto teórico de la cadena agroalimentaria para estudiar las interrelaciones de la agricultura con otras actividades y analizar su peso específico en el sistema agroalimentario. Es una esquematización de la secuencia vertical de las etapas de producción/transformación/distribución del conjunto del sistema agroalimentario, tanto en lo que respecta a las empresas pertenecientes al sistema agroalimentario como a las vinculadas económicamente al mismo mediante relaciones de compra-venta de bienes o de prestación de servicios. Se hace hincapié en la aportación de cada una de las etapas a la formación del producto de consumo final, en términos tales como flujos de cantidades físicas, de valor de mercado, de energía, de trabajo o de capital (Sanz, 1997).

Porter (2010) introduce el concepto de cadena de valor para estudiar las fuentes que suponen una ventaja competitiva para las empresas, señalando que se trata de una herramienta básica para examinar todas las actividades que la empresa realiza y su manera de interactuar. La cadena de valor permite dividir a la empresa en sus actividades estratégicamente relevantes a fin de entender el comportamiento de los costes, así como las fuentes actuales y potenciales de diferenciación. La ventaja competitiva se logra cuando la realiza mejor o con menor coste que los competidores.

La salida de la crisis de los años setenta provocó la reestructuración del sistema agroalimentario intensificando las relaciones de espacios geográficos distantes y la integración de los mercados nacionales, dando paso a lo que se conoce como globalización (Koc, 1994). El sistema agroalimentario se organiza a nivel mundial con provisión de inputs en todo el mundo, para la producción de alimentos a nivel global. Las industrias alimentarias transnacionales, y en particular las grandes empresas de la distribución comercial, tienen una alta capacidad de influir en los hábitos 
de consumo de la población, controlando el tipo de producto que se presentan en el mercado, aislando al productor agrario de la demanda final (Etxezarreta, 2006).

Además, la empresa en red se ha convertido en un elemento central de las relaciones entre los diferentes agentes. Se define como una estructura sinérgica, que articula contractualmente, a medio plazo, relaciones interempresariales, a fin de responder conjunta y solidariamente, de manera flexible, bajo la dirección de una empresa donadora de órdenes, a una demanda final o intermediaria volátil. La logística tiene una creciente importancia con la puesta en marcha de redes de circulación de información, entre los distintos operadores, con sistemas informáticos comunes. Así, el recurso a la información permite disminuir el coste de movimientos innecesarios de mercancías, que se desplazan sólo en el momento y hacia el lugar necesario (Green y Dos Santos, 1992).

Estamos asistiendo, por lo tanto, a la implantación de redes mundiales de producción, o como algunos prefieren llamarlo, a redes de producción transnacional. La principal causa de esta mayor dispersión geográfica ha sido la revolución en las tecnologías de las comunicaciones y los transportes (Dicken, 2007).

La dispersión global de la producción y el consumo de los productos sugiere el concepto de la cadena productiva global, pues facilita la comprensión de la estructura y la dinámica de las industrias globales. Gereffi (2001) introduce la dimensión internacional en el análisis de cadena productiva y estudia la gobernanza de la cadena, identificando a las empresas que ejercen el poder en los diferentes segmentos de la cadena productiva y contempla la coordinación como una fuente clave para conseguir una ventaja competitiva. Y diferencia entre cadenas productivas dirigidas al productor que son aquellas en las que los grandes fabricantes transnacionales juegan los papeles centrales en la coordinación de las redes de producción, y las cadenas productivas destinadas al comprador que se refieren a aquellas en las que son los grandes detallistas, comerciantes y fabricantes de marca, los que ejercen los roles centrales.

El concepto de gobernanza es un punto central en el análisis de las cadenas agroalimentarias. El término gobernanza se refiere a las diferentes formas de control/ coordinación que las empresas líderes ejercen en la cadena de valor global. De tal modo que, las empresas líderes influyen directamente o indirectamente en la organización de la producción, en la logística y comercialización de los productos. Esta influencia puede extenderse a la definición de los productos, los procesos y los estándares que utilizan las empresas que integran la cadena específica (Gereffi et al., 2001).

Humphrey y Schmitz (2004) distinguen cuatro tipos de relaciones en la cadena de valor global:

- De mercado entre agentes independientes, el comprador y el vendedor no desarrollan relaciones cercanas o de colaboración porque el producto es estándar. Esto implica que los requisitos de los compradores pueden ser satisfechas por una serie de empresas-proveedores y que los costes de cambio para ambas partes son bajos.

- Las empresas configuran redes, intercambian la información y dividen las competencias de la cadena de valor entre ellas. Las relaciones se caracterizan por la coordinación y la dependencia reciproca.

- Cuasi jerárquicas. Una empresa ejerce un alto grado de control sobre otras empresas de la cadena, especificando las características del producto a fabricar, y a veces especifica los procesos a seguir y los mecanismos de control que deben cumplir.

- Jerárquica. La empresa principal tiene la propiedad directa de algunas operaciones en la cadena ejerciendo un importante control de los productos y los procesos. 
La información en las decisiones empresariales

La principal característica de la administración de la empresa es convertir la información en acción, a través de un proceso de decisión. De este modo, la relación información-decisión-acción representa el eje central de todo el sistema de dirección. El proceso de decisión puede estudiarse sobre la base de una matriz en la que se recogen los sucesos posibles o estados del entorno, las probabilidades de la ocurrencia de éstos, las estrategias o alternativas y los resultados. Sobre esta matriz se desarrollará el proceso de decisión en función del grado de conocimiento del entorno, de la verosimilitud en la estimación de los resultados y, en definitiva, de las características de la información disponible (Bueno-Campos, 2004).

Además, la adopción de decisiones se halla condicionada por un amplio conjunto de factores de diversa naturaleza, entre los que pueden destacarse: factores de carácter estrictamente objetivo, como son los que representan la estructura básica del problema que requiere la decisión adoptar; factores de carácter subjetivos, que se encuentran íntimamente asociados al grado de conocimiento por parte de sujeto del problema presentado y de su entorno; y los factores institucionales, tales como el tipo de leyes sociales vigente en la comunidad, el ordenamiento jurídico, etc. (López-Cachero, 1989). De este modo, la toma de decisiones resulta muy compleja, y exige un alto nivel de información sobre los factores más influyentes y con mayor repercusión.

El proceso de decisión tiende a buscar la mejor opción posible. Es evidente que la solución óptima está condicionada por el riesgo de las variables de decisión y por la incertidumbre originada por el grado de conocimiento o de información supuestos en la decisión. Por consiguiente, la decisión puede plantearse en función del riesgo e incerti- dumbre a que se somete. Según el contenido estimado de aleatoriedad y de información, se establecen tres modelos de decisión. En primer lugar, el modelo determinista, que sigue el método de las ciencias exactas, apoyándose en mediciones precisas y cálculos matemáticos. De este modo, la aleatoriedad de las variables de decisión se reduce a cero. En oposición al modelo matemático determinista y en función del elemento aleatorio, se encuentra el modelo probabilístico, a veces Ilamado estocástico. El concepto de probabilidad es cuestionable cuando se intenta aplicar a la economía, ya que el cálculo de la probabilidad no está exento de objeciones y la interpretación que puede hacerse de la probabilidad matemática es siempre discutible. El tercero es el modelo de información, que sugiere que las decisiones están determinadas por las condiciones que determinan el éxito o el fracaso de los resultados o consecuencias de la decisión. Esto se conoce como estado de naturaleza, comprendiendo el conjunto de circunstancias o características que particularizan la decisión. Por consiguiente, la información del estado de naturaleza describe el estado del problema según el contexto en que actúa la decisión. El modelo de información precisamente analiza la toma de decisiones según la información que se tiene sobre los estados de naturaleza (Soldevilla-García, 1984).

Esta investigación constituye una aproximación al estudio de los modelos de información, pues se centra en detectar las necesidades de información de los productores fundamentalmente y plantea una herramienta para mejorar el proceso de toma de decisiones en la comercialización de sus productos.

\section{Metodología}

La investigación empleó fuentes primarias fundamentalmente, centradas en la realización de entrevistas a los agentes relevantes (productores, productores-exportadores, ex- 
portadores y asociaciones) de la cadena de valor del sector de los cítricos en Andalucía. Entre las fuentes bibliográficas y documentales, destacamos los estudios realizados recientemente por instituciones autonómicas sobre el sector de los cítricos en Andalucía: Consejería de Agricultura y Pesca (2009) y Analistas Económicos de Andalucía (2011). La principal aportación de esta investigación es la identificación de las necesidades de información que tienen los agentes de la cadena de valor de los cítricos, las posibilidades que tendría la implantación de una plataforma virtual para mejorar sus decisiones empresariales en la venta de los productos, las áreas temáticas más valoradas y el modelo de gestión que debería seguir la plataforma.

Para cumplir el objetivo principal de la investigación, se diseñó un cuestionario que se estructuró en nueve partes. La primera tenía como meta conocer los rasgos básicos del agente entrevistado y sus actividades productivas. Con la segunda y tercera se perseguía conocer las desventajas competitivas de los agentes en la cadena de valor, y las necesidades de información sobre el nivel de precios en cada uno de los canales de comercialización de los productos, respectivamente. Entre los capítulos cuarto y octavo, se incluyeron una serie de preguntas con las que se trataba de conocer las necesidades de información sobre los temas siguientes: operaciones financieras, transporte y logística, aduanas y legislación, seguridad alimentaria y las acciones de promoción del consumo de cítricos. La última parte del cuestionario tenía como finalidad conocer la disposición que presentan los agentes de la cadena de valor para remunerar los servicios que les suministraría la plataforma virtual e identificar la naturaleza (pública o privada) de la entidad gestora.

En el primer semestre de 2012 se realizaron 32 entrevistas personales a agentes de la cadena de valor entre los que se encontraban los principales productores y asociaciones de productores de Sevilla, Huelva y Córdoba, así como la Federación de cooperativas que representan $65 \%$ de la producción de cítricos en Andalucía, y las empresas comercializadoras más importantes con el $50 \%$ de la exportación total. Se trata por tanto de una muestra con una importante representatividad en el conjunto del sector de los cítricos en Andalucía. También se entrevistó al responsable Frutas y Hortalizas del SOIVRE (Servicio Oficial de Inspección, Vigilancia y Regulación del Comercio Exterior) que se encarga del control del comercio exterior de productos agroalimentarios. Los agentes locales y expertos entrevistados comprenden las siguientes tipologías sobre la base de la forma jurídica: 9 Cooperativas de primer grado, 2 Cooperativas de segundo grado, 7 Sociedades Agrarias de Transformación (SAT), 5 Sociedades Limitadas (SL), 3 Sociedades Anónimas (SA), 4 Asociaciones de productores (AP), un Grupo de Desarrollo Rural y el Servicio Oficial de Inspección y Vigilancia Aduanera. Para el análisis estadístico de los datos obtenidos con la encuesta se ha utilizado el paquete informático SPSS (2006).

\section{Resultados y discusión}

Caracterización de la población encuestada

Como se ha comentado en material y métodos, la población encuestada consistió en 32 agentes de la cadena de valor de cítricos en Andalucía, correspondientes a diversas tipologías jurídicas.

En cuanto al tipo de cítrico que producen las empresas entrevistadas, destaca la producción de naranja, relacionada con el espectacular crecimiento de la superficie cultivada de naranjo dulce que en los últimos años se ha producido en las principales zonas productoras andaluzas, y mandarina, ambas claramente superiores a la de otros cítricos (limón, po- 
melo, etc.). La producción comercializada por las entidades de tipo asociativo (Sociedades Cooperativas Andaluzas SCA y Sociedades Agrícolas de Transformación SAT) es mayoritariamente de sus socios, mientras que las Sociedades Anónimas producen y venden su propia cosecha, aunque también comercializan fruta de terceros, y en las Sociedades Limitadas tiene cierta relevancia la compra a agricultores de la zona. No obstante, en los últimos años, las empresas encuestadas reconocen un aumento de las importaciones de cítricos procedentes de otros países del Mediterráneo y del Hemisferio Sur (especialmente Sudáfrica), lo que está provocando un descenso de los precios.

La mayoría de las empresas encuestadas comercializan más del $75 \%$ de la fruta en fresco y el resto a la industria del zumo. La produc- ción de IV y $V$ gama, y de otros transformados tiene una escasa participación en las ventas totales. Según la forma jurídica, excepto las SCA, que diversifican en mayor proporción la producción ( $61 \%$ en fresco, $37 \%$ de zumos y $2 \%$ de $V$ gama), las demás empresas comercializan en fresco más del $80 \%$ de la fruta y el resto lo destinan a la producción de zumos (Figura 1). La Unión Europea es el principal destino de las exportaciones de cítricos aunque las empresas encuestadas reconocen que probablemente una gran parte de la producción es exportada a través de la Comunidad Valenciana. Esto supone una importante merma de la rentabilidad de las empresas andaluzas y la necesidad de mejorar sus estructuras comerciales. En general, las empresas disponen de al menos un técnico comercial para la venta de la producción. Mientras que las entidades privadas cuentan con personal

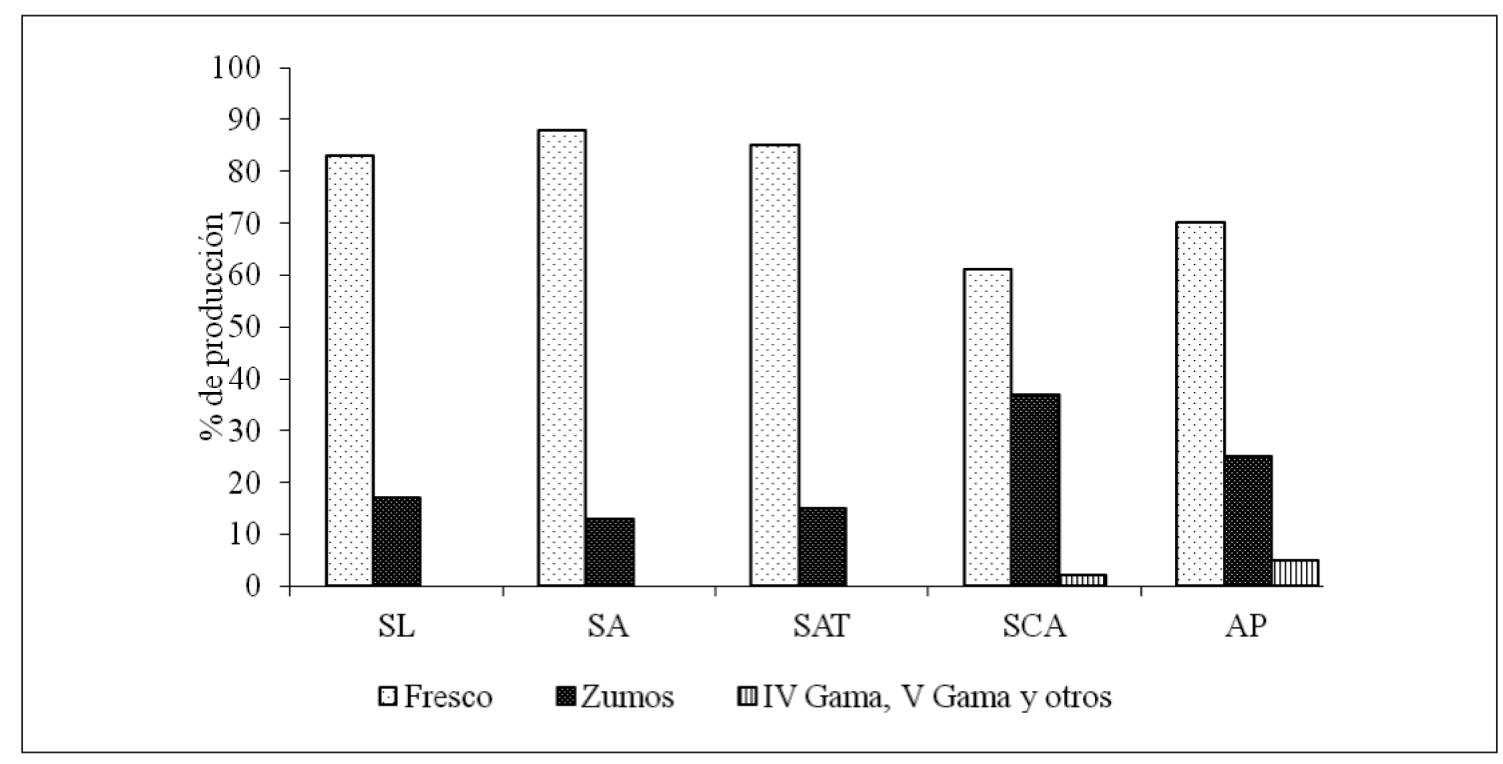

Fuente. Elaboración propia a partir de la encuesta realizada en 2012.

Figura 1. Destino de la producción comercializada según la forma jurídica de las entidades. S.L.: Sociedad Limitada; S.A.: Sociedad Anónima; SAT: Sociedad Agraria de Transformación; SCA: Sociedad Cooperativa; AP: Asociación de Productores.

Figure 1. Products destination according to the legal nature of the entity. 
especializado para la comercialización de la producción, en las entidades asociativas es muy frecuente que el gerente desarrolle tanto tareas administrativas como comerciales.

Los productores entrevistados venden sus productos en tres canales: a la industria y almacenistas/exportadores, que reciben el $43 \%$ de las ventas totales cada uno, y a las SCA (Sociedad Cooperativa) de primer y segundo grado, que reciben el $14 \%$ restante (Figura 2 ). El exportador vende su producción en tres canales que tienen la misma representatividad: el $34 \%$ a la industria, $33 \%$ a centrales o plataformas de compras en origen y el restante $33 \%$ se vende a los grandes supermercados o cadenas en el extranjero.

Los productores-exportadores tienen una gran variedad de canales para vender su producción, destacando la industria con el $24 \%$ de las ventas totales, los grandes supermercados o cadenas extranjeras con el $18 \%$, los almacenistas/exportadores con el $16 \%$, los grandes supermercados o cadenas nacionales con el $13 \%$, las centrales o plataformas de compra en el exterior con el $13 \%$, las centrales o plataformas de compra en origen con el $10 \%$, las ventas directas con el $4 \%$ y los canales alternativos y las cooperativas de primer y segundo grado con el $1 \%$ cada uno de ellos. Las asociaciones encuestadas manifiestan que la producción de cítricos en Andalucía se comercializa del siguiente modo: $25 \%$ a la industria, $25 \%$ a centrales o plataformas de compra en el exterior, $13 \%$ a SCA de primer y segundo grado, $13 \%$ canales alternativos, $12 \%$ a almacenista/exportador y $12 \%$ a gran supermercado o cadena nacional. El comercio electrónico (webs, venta por teléfono, etc.) tiene una escasa importancia en las ventas totales, probablemente por la falta de confianza en este tipo canales no relacionales, y no se producen ventas al canal HORECA (HOstelería, REstauración, CAterings). Se trata por tanto, de importantes nichos de mercado que probablemente mejorarían la rentabilidad de los productores.
Necesidades de información

En relación a las temáticas consultadas, los encuestados señalan que tienen altas necesidades sobre la "Organización de la Oferta" y la "Cadena de valor de los cítricos" y los "Mecanismos de formación de los precios de venta", indicando que la fuente de información más utilizada para conocer la cadena de valor de los cítricos es la fuente relacional (clientes y empresas del sector), con un alto nivel de confianza. Además, los agentes indican que el Observatorio de Precios en Origen y Destino de la Consejería de Agricultura, Pesca y Desarrollo Rural de la Junta de Andalucía y el Panel de Consumo del MAGRAMA son fuentes de información poco examinadas por los agentes de la cadena de valor para conocer los precios de los productos cítricos. Una posible explicación sería que las fuentes públicas no permiten obtener una información en tiempo real sobre los niveles de precios para favorecer sus operaciones comerciales. Esto dificulta el nivel de eficiencia del proceso de toma de decisiones empresariales de los productores y perjudica seriamente su nivel de rentabilidad.

Por lo que se refiere a la "Seguridad de las operaciones financieras", los encuestados señalan que necesitan más información sobre la fiabilidad y solvencia de sus clientes, porque de ello depende el cobro de sus ventas y también la posibilidad de asegurarlas. Sus principales fuentes de información son las empresas de seguros. En cuanto al "Transporte y la Logística", los encuestados tienen unas necesidades medias o altas, en temas como la solvencia, puntualidad de las empresas transportistas y seguros asociados. En materia de "Legislación y aduanas", los encuestados muestran unas necesidades altas, pero los servicios de los que dispone la Administración no resultan atractivos para ellos, pues la mitad de los entrevistados no conoce los protocolos de internacionalización de las empresas españolas del Instituto de Comer- 


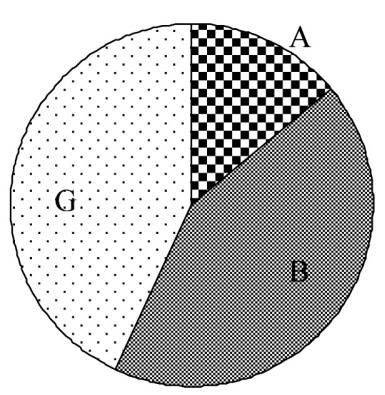

Productores

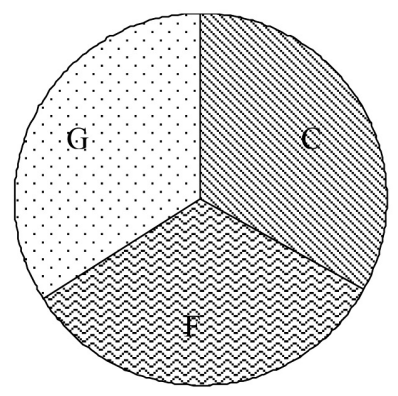

Exportadores

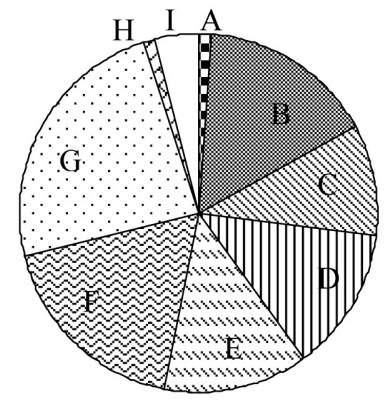

Productores-Exportadores

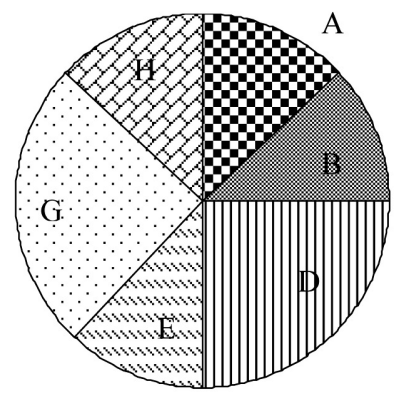

Asociaciones

A: Cooperativas de primer y segundo grado

B: Almacenistas exportadores

Q $\mathrm{C}$ : Centrales o plataformas de compra en origen

巴 D: Centrales o plataformas de compras en el exterior

Q E: Gran superficie o cadena nacional

슬 F: Gran superficie o cadena en el extranjero

$\square \mathrm{G}$ : Industria

H: Canales alternativos

口I: Venta directa

Fuente. Elaboración propia a partir de la encuesta realizada en 2012.

Figura 2. Relaciones con otros agentes de la cadena de valor según la actividad de los agentes. Figure 2. Relationships with other Value Chain agents according to their activity. 
cio Exterior (ICEX) y Extenda (Agencia Andaluza para el comercio exterior), ni consulta las bases del ICEX sobre productos fitosanitarios y residuos permitidos en cítricos.

Además, se detectan altas necesidades de información en la implantación de los sistemas de "Trazabilidad y seguridad alimentaria". Sólo el $83 \%$ de los agentes encuestados tiene implantado un sistema de trazabilidad básico y obligatorio (debería ser del $100 \%$ ), el $83 \%$ dispone del sistema de Autocontrol y el $50 \%$ tiene el sistema APPCC (Análisis de Peligros y Puntos de Control Críticos) (Figura 3). Asimismo, el nivel de implantación de las normas privadas de certificación de la calidad es muy superior al de las públicas. Entre las normas privadas hay un claro liderazgo de GlobalGAP (el $96 \%$ de los encuestados la tienen implantada en campo y el $79 \%$ en almacén). Destacan también las normas BRC (British Retail Consortium), IFS (International Food Standard) y a más distancia otros protocolos como Nature Choice. Entre las normas públicas, las más frecuentes son las certificaciones de calidad de Producción Integrada y Producción Ecológica. Las razones indicadas por los agentes entrevistados para implantar dichos protocolos de certificación son: la imposición del cliente, el aumento de las posibilidades de venta $y$, en menor medida, el incremento de calidad, pero en ningún caso los asocian a un mayor precio del producto. Para los distribuidores, estos sellos de calidad certificada son una garantía de que los alimentos cumplen con los niveles establecidos de calidad y seguridad, y de que se han elaborado siguiendo criterios de sostenibilidad, respetando la seguridad, higiene y el medio ambiente, lo que reduce los costes de transacción entre los productores y distribuidores.

Grazia y Hammoudi (2012) distinguen dos sistemas de seguridad alimentaria: los instrumentos públicos de regulación para asegurar la verificación del cumplimiento de la legislación sobre piensos y alimentos y toda una serie de estándares privados, bien "internos" de cada empresa o en el contexto de las relaciones de compradores-proveedores, especialmente impuestos por los grandes minoristas de alimentos, fabricantes y operadores de servicios para acceder al mercado.

El grado de conocimiento de los "Hábitos de consumo de los cítricos" es en general mediobajo, con claras diferencias entre los diferentes tipos de mercado: hay un mayor desconocimiento del mercado local o nacional que del mercado internacional, lo que pone de manifiesto la vocación exportadora del sector de cítricos. Las fuentes de información más utilizadas por los agentes encuestados son los viajes de prospección de mercados y los Informes elaborados por las Interprofesionales, así lo indican el $37 \%$ y $34 \%$ de los encuestados, respectivamente. Le siguen el Observatorio del Consumo y la Distribución Alimentaria (OCDA) del MAGRAMA, indicado por el $27 \%$ de los encuestados, y los Informes del ICEX, por el $23 \%$. Además, el $23 \%$ consultan otras fuentes (Informes de FAECA, Asociafruit, clientes, etc.) (Figura 4). De este modo, las fuentes de información de organismos oficiales, como el MAGRAMA o el ICEX están peor valoradas que las fuentes privadas: viajes de prospección o encuestas propias. Además, el tema de la promoción y el consumidor final despierta gran interés entre los agentes encuestados, los cuales demandan información sobre ayudas directas a la promoción, canales de promoción de bajo coste, estructura comercial de potenciales países de destino, tendencias de consumo de fruta fresca y zumos, datos de consumo detallado a nivel varietal y por países, etc.

\section{Potencialidad de la plataforma} de información

La población encuestada está familiarizada con las herramientas informáticas puesto que usa frecuentemente tecnologías TIC para la búsqueda de información, fundamentalmente 


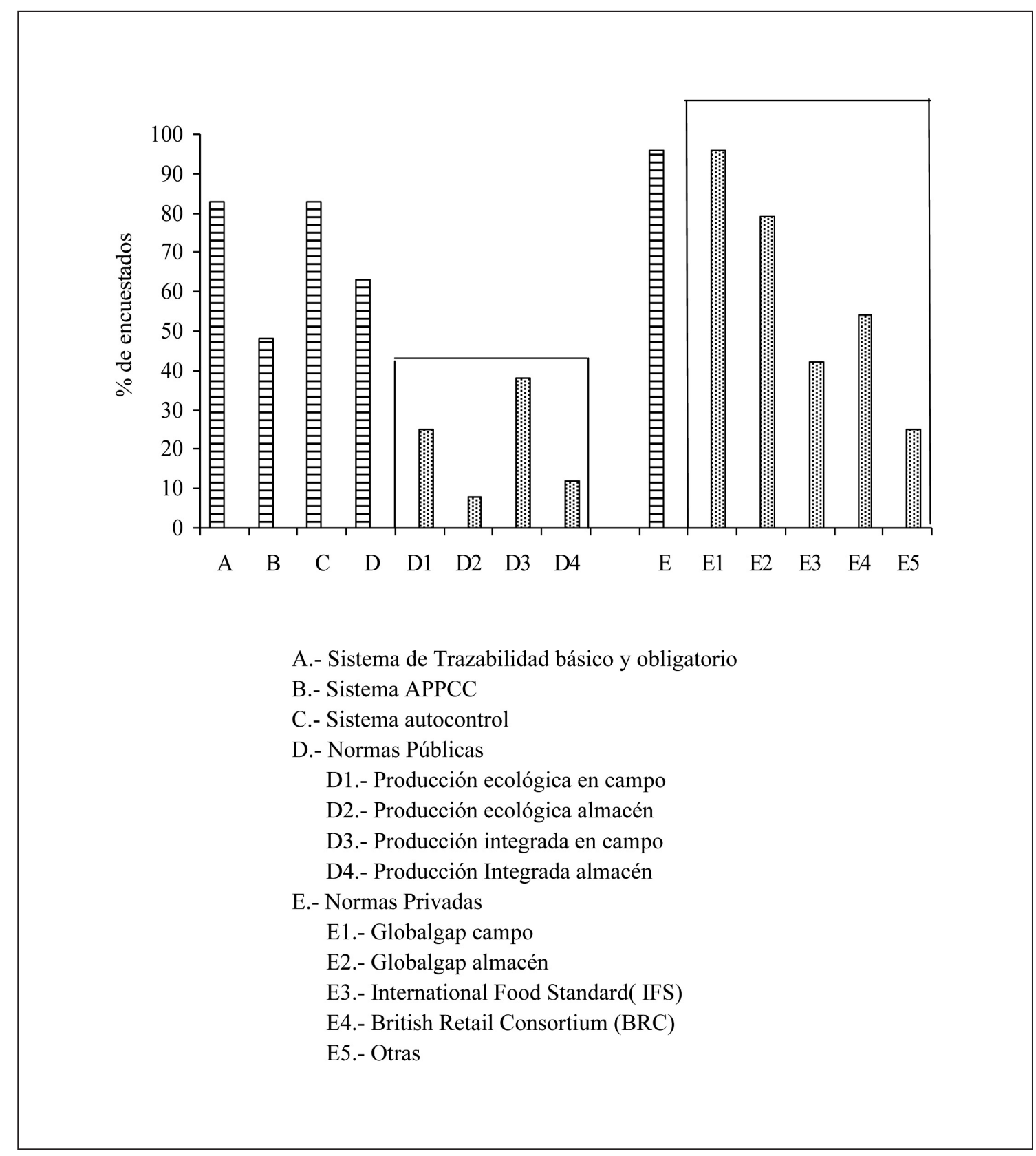

Fuente. Elaboración propia a partir de la encuesta realizada en 2012.

Figura 3. Sistema de trazabilidad implantado.

En barras con línea horizontal se presentan los principales niveles de trazabilidad. Los niveles dentro de las Normas públicas y privadas están detallados en las barras de puntos. Figure 3. Traceability system implemented. 


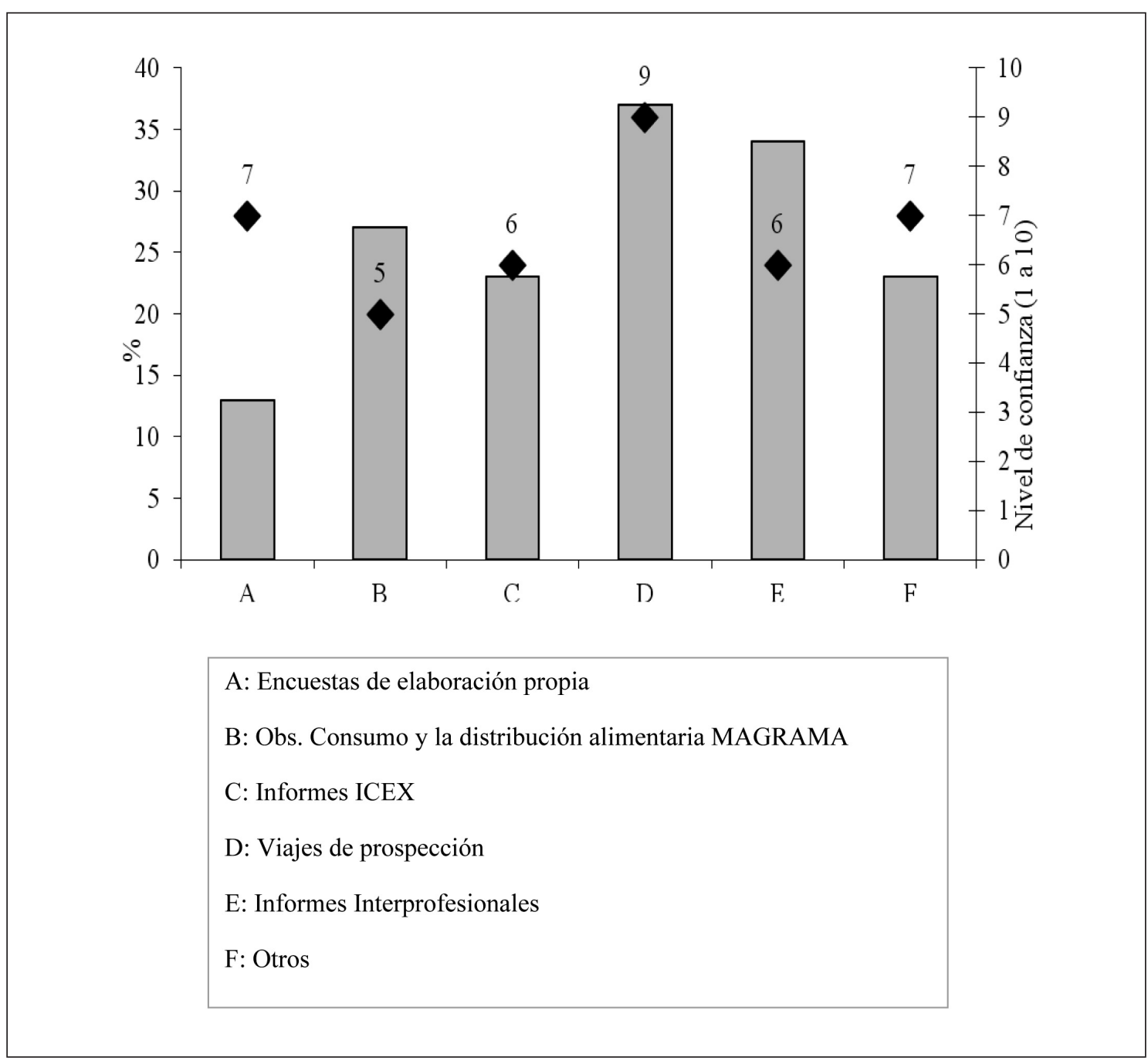

Fuente. Elaboración propia a partir de la encuesta realizada en 2012.

Figura 4. Fuentes de información y nivel de confianza en el tema de "Promoción y hábitos de consumo".

Figure 4. Information Sources and trust level with regard to the topic "Promotion and consumption habits".

Internet a través del ordenador (100\%) y además a través del móvil (87\%), aunque el uso de las redes sociales y los foros es menor ( $36 \%$ y $23 \%$, respectivamente). Entre las notables potencialidades que tendría la plataforma de información destacan la posibilidad de compartir información en tiempo real y el rápido acceso al conocimiento, lo que favorecería la eficiencia en la toma de decisiones y la coordinación de las acciones de los productores. 
Los agentes encuestados indican que las temáticas prioritarias que debe contener la plataforma son: la Organización de la oferta y estructura de la cadena de valor de los cítricos, Mecanismos de formación de precios y Promoción y consumidor final, Transporte y logística, Legislación y aduanas, y Trazabilidad y seguridad alimentaria. Los productores demandan más información agronómica que de otros aspectos, sin embargo la mayoría no conocen los productos que demanda el consumidor final. Cuando proyectan una nueva plantación de cítricos, consultan al viverista y a otros productores, pero lo lógico sería deducir esa información de las necesidades de sus futuros compradores.

Los agentes, entre ellos todos los productores y el único exportador, estarían dispuestos a compartir información sobre precios y clientes. No obstante, el $63 \%$ ponen algún tipo de condicionante, como "solo con empresas muy relacionadas con el sector", "condicionado a que la plataforma funcione bien", etc.

\section{Propuestas}

Los agentes manifiestan ciertas diferencias en la ordenación de prioridades de información en la hipotética plataforma virtual. Los productores valoran fundamentalmente las temáticas que afectan a su actividad y precio, especialmente la Organización de la oferta y estructura de la cadena de valor y los Mecanismos de formación de precios (valoración media 9). Las temáticas menos valoradas son la Legislación y aduanas y Promoción del consumidor final (valoración media 2) posiblemente porque están especializados en la etapa productiva y no se dedican al comercio exterior ni a la comercialización del producto final (Figura 5). El resto de agentes manifiestan un importante interés por todas las temáticas (valoración media mayor o igual a 6) y de forma mucho más homogénea.

El 73\% de los encuestados estarían dispuestos a pagar por la información de la plata- forma, mientras que un $20 \%$ tiene claro que no pagaría y el 7\% NS/NC. De los que contestan afirmativamente, el $68 \%$ pondría restricciones a dicho pago, fundamentalmente en función del "tipo de información y precio" y puntualmente un encuestado estaría dispuesto a pagar "en función de la calidad del servicio". Las diferencias entre las actividades de los agentes suponen distintas disposiciones para remunerar la información. Todos los productores y exportadores estarían dispuestos a retribuir la información que suministraría la plataforma y el $24 \%$ de los productores-exportadores y el $33 \%$ de las asociaciones no pagarían por ello (Figura 6).

La mayoría de los agentes piensa que la plataforma debe ser gestionada de forma mixta, entre la Administración pública y empresas o asociaciones privadas. Creen, por su experiencia, que si la plataforma es gestionada por la Administración, no llegaría a ser una plataforma dinámica y viva, y por el contrario si fuera exclusivamente gestionada por una empresa o asociación, ésta la intentaría utilizar para su propio beneficio o el de sus socios. Se propone, por ejemplo, que sea la Administración quien desarrolle la plataforma, y que los agentes le den vida. Deberían también participar organismos públicos de investigación, como la Universidad y el IFAPA, utilizando esta plataforma para informar a los agentes de los estudios y los proyectos en curso.

\section{Conclusiones}

Los agentes encuestados en este trabajo son representativos del sector citrícola de Andalucía occidental, particularmente de las provincias de Sevilla y Huelva. Fundamentalmente producen naranjas y mandarinas, y comercializan su producción en fresco, siendo su principal destino el mercado exterior, sobre todo países de la Unión Europea. En su mayoría se trata de SCA y SAT, con una media de 10 trabajadores fijos y 100 eventuales. 

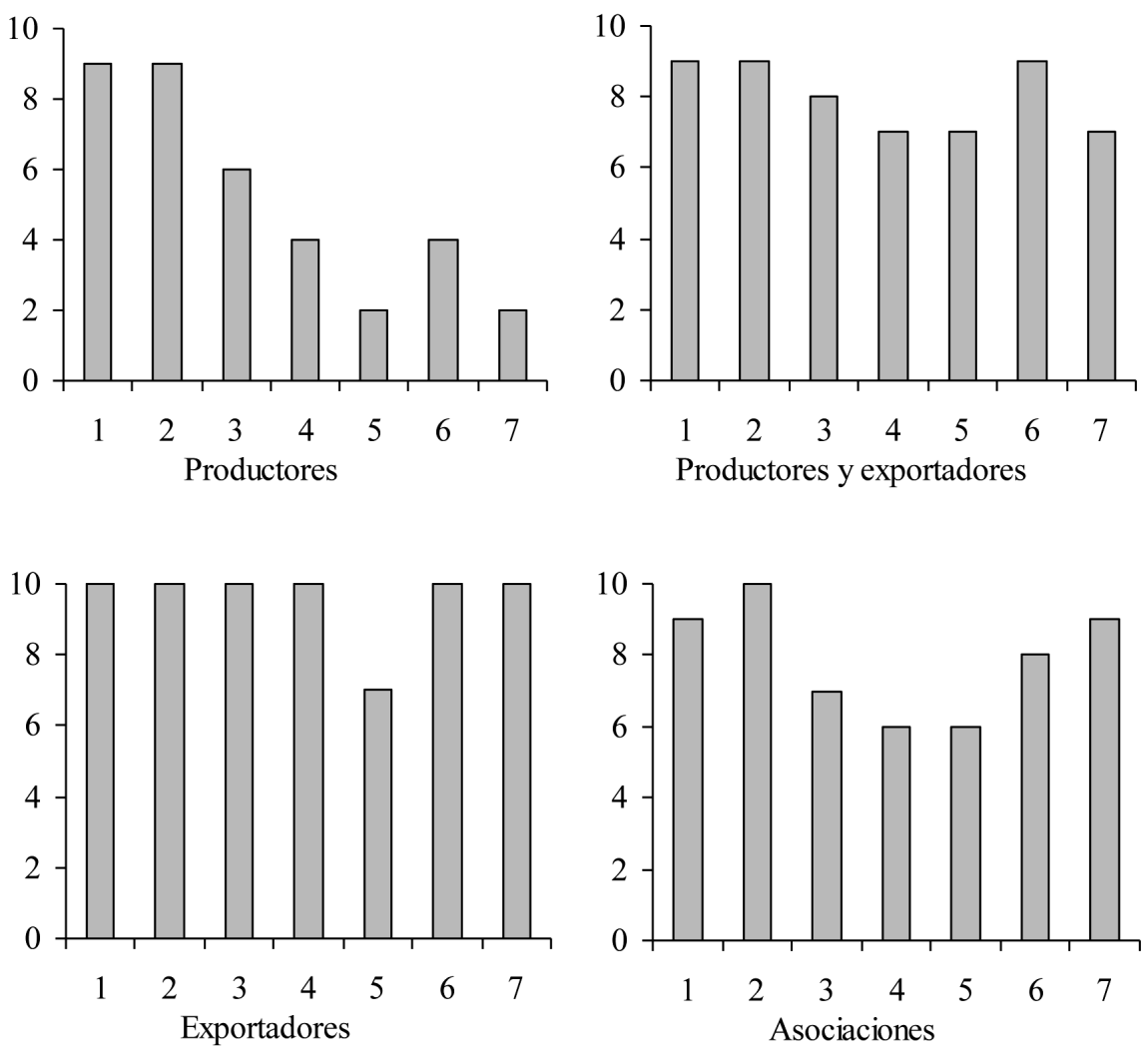

1. Organización de la oferta y estructura de la cadena de valor

2. Mecanismos de formación de precios

3. Seguridad de las operaciones financieras

4. Transporte y logística

5. Legislación y aduanas

6. Trazabilidad y seguridad alimentaria

7. Promoción y consumidor final.

Fuente. Elaboración propia a partir de la encuesta realizada en 2012.

Figura 5. Preferencias (1 a 10) de los agentes por las diferentes temáticas para la hipotética plataforma virtual.

Figure 5. Preferences of the agents for the different subject matters for the hypothetical virtual platform. 


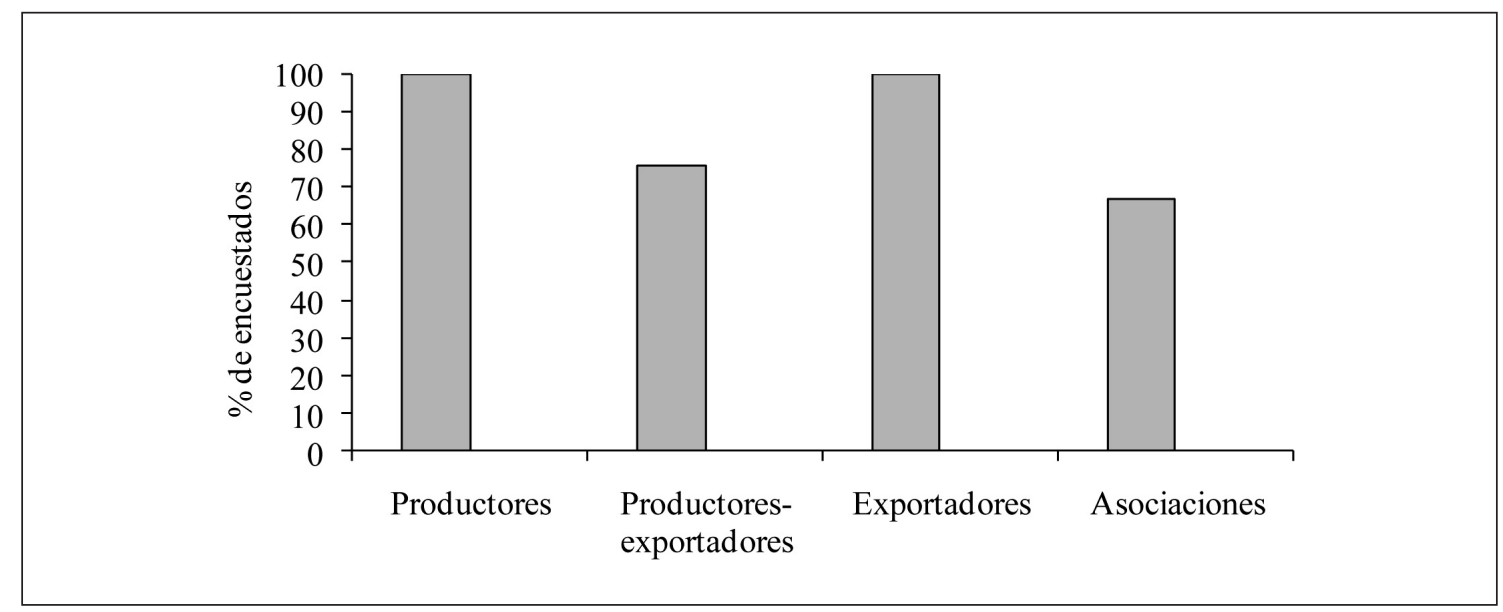

Fuente. Elaboración propia a partir de la encuesta realizada en 2012.

Figura 6. Disposición a pagar por la información en la futura plataforma según actividad de los agentes.

Figure 6. Willingness to pay for the information provided by the potential platform according to the activity of the respondent.

El proyecto de implantación de una plataforma de información en el sector de los cítricos en Andalucía puede ser viable puesto que hay una clara demanda de información por parte de la población encuestada, que supone el $65 \%$ de la producción citrícola andaluza y el $50 \%$ de la exportación total. La demanda es clara en todo lo relacionado con la organización de la oferta y estructura en la cadena de valor de los cítricos, mecanismos de formación de precios y promoción y consumidor final. Se advierte una gran necesidad de información sobre los niveles de los precios. Algunos agentes señalan que sería interesante especificar precios según variedades, calibres y tipos de envases de los productos vendidos a la industria.

El modelo de plataforma debería ser de gestión mixta, público-privada. La Administración se encargaría de implantar la herramienta y del control de la información, y los agentes le aplicarían un carácter dinámico y vivo. También deberían participar organismos públicos de investigación como la Universidad y el IFAPA
(Instituto Andaluz de Investigación y Formación Agraria y Pesquera), que informarían de los resultados de sus trabajos de investigación. La mayoría de los encuestados estarían dispuestos a pagar por los servicios que suministre la plataforma, si bien indican algunas limitaciones en cuanto al acceso, calidad del servicio y precio.

\section{Agradecimientos}

Esta investigación se ha desarrollado por encargo de la Fundación Corporación Tecnológica de Andalucía (CTA), a través del Contrato de Investigación 1480/0565, con el fin de utilizar los resultados para su proyecto Trading. Los autores quieren expresar su agradecimiento a los agentes sociales entrevistados por su colaboración en el desarrollo de esta investigación y a Mariana Sánchez Ocaña por su apoyo técnico. 


\section{Bibliografía}

Analistas Económicos de Andalucía (2011). Competencia y Cadena de valor en la producción y distribución de cítricos. Unicaja, Málaga. 134 pp.

Arenas F, Carmona A, Salguero A, Hervalejo A, Martínez-Ferri E, Alfonso JM (2009). El cultivo de los cítricos en Andalucía. Levante Agrícola 397: 272-278.

Arenas F, Castillo C, Hervalejo A (2011). Continúa aumentando la producción de cítricos en Andalucía con nuevas variedades tardías. Vida Rural 328: 40-44.

Arenas F, Hervalejo A (2013). Análisis del cultivo de los cítricos en Andalucía. Vida Rural 362: 34-40.

Aznar-Sánchez JA, Galdeano-Gómez E, ÁlvarezRamos J, Tapia-León J, Godoy A (2013). El sector de la comercialización hortícola en Almería. Concentración, prospectiva y logística. Cajamar, Serie Economía, Almería. 121 pp.

Bueno-Campos E (2004). Curso básico de Economía de la Empresa. Un enfoque de organización. Ediciones Pirámide, Madrid. 710 pp.

Consejería de Agricultura, Pesca y Desarrollo Rural (CAPDR) (2013). Anuarios de Estadísticas Agrarias y Pesqueras de Andalucía. Disponible en http://www.cap.junta-andalucia.es (03 abril 2014).

Consejería de Agricultura y Pesca (2009). Análisis del sector citrícola en Andalucía. Dirección General de Planificación y análisis de mercados. Consejería de Agricultura y Pesca, Junta de Andalucía, Sevilla. 66 pp.

Delgado C (2013). La cesta doblega al carrito. El País. 10 de noviembre.

Dicken P (2007). Global Shift. Mapping the changing contours of the world economy.Sage Publications, London. 632 pp.

Eraso E (2010). Análisis de la comercialización del sector de los cítricos en Andalucía. En. Analistas Económicos de Andalucía Informe sectorial Agrario en Andalucía 2009. Málaga: 303-320.

Etxezarreta M (2006). Los elementos que conforman la evolución de la agricultura. En: La agri- cultura española en la era de la globalización (Ed. Etxezarreta M), pp. 27-213. Ministerio de Agricultura, Pesca y Alimentación, Madrid.

FAO, 2013. Organización de las Naciones Unidas para la Alimentación y la Agricultura. Food and Agriculture Organization of the United Nations. Disponible en http://faostat.org (14 marzo 2013)

Gereffi G (2001). Las cadenas productivas como marco analítico para la globalización. Problemas del Desarrollo 125: 9-37.

Gereffi G, Humphrey J, Kaplinsky R, Sturgeon T (2001). Introduction: Globalisation, Value Chains and Development. Institute of Development Studies, Bulletin 32.3. 12 pp.

Grazia C, Hammoudi A (2012). Las cadenas de valor globales y la heterogeneidad en los estándares de seguridad alimentaria: una panorámica sobre las principales cuestiones económicas. Cuadernos de Estudios Agroalimentarios 04: 47-68.

Green RH, Dos Santos R (1992). Economía de Red y reestructuración del sistema agroalimentario. Estudios Agro-Sociales 162:37-61.

Humphrey J, Schmitz H (2004). Chain governance and upgrading: taking stock. In: Local enterprises in the global economy (Ed. H Schmitz), pp. 349-381. Massachusetts, USA.

Koc M (1994). La globalización como discurso. En: Globalización del sector agrario y alimentario (A Bonanno), pp. 51-78. Ministerio de Agricultura y Pesca y Alimentación. Madrid, España.

Langreo A (2012). La estrategia de la gran distribución y su incidencia en la cadena producción. Cuadernos de Estudios Agroalimentarios 4: 29-46.

López-Chachero M, (1989). Análisis y adopción de decisiones. Ediciones Pirámide, Madrid.447 pp.

MAGRAMA (2013) Ministerio de Agricultura, Alimentación y Medio Ambiente. Anuario de Estadística 2010. Disponible en http://www.magrama.gob.es. (14 marzo 2013).

Mili S (1996). Organización de mercados y estrategias empresariales en el subsector del aceite de oliva. Ministerio de Agricultura y Pesca y Alimentación, Madrid. 390 pp. 
Ministerio de Industria, Turismo y Comercio (2010). La distribución comercial en España. Boletín económico de ICE 2993: 9-223.

Porter M (2010). Ventaja Competitiva. Creación y sostenibilidad de un rendimiento superior. Pirámide, Madrid. 592 pp.

Rebollo-Arévalo A (2012). Evolución de los márgenes comerciales de los productos frescos de alimentación. Distribución y Consumo 122: 1-13.

Sanz J (1997). El Sistema Agroalimentario Español. Cambio estructural, poder de decisión y organización de la cadena agroalimentaria. En:
Agricultura y sociedad en la España contemporánea (Ed. J Gómez C y González), pp. 355396. CIS, Madrid.

Soldevilla-García E (1984). Decisiones empresariales con riesgo e incertidumbre. Editorial Hispano Europea, Barcelona. 473 pp.

SPSS Inc. (2006). Manual del Usuario de SPSS Base 15.0. SPSS Inc., Chicago. 177 pp.

(Aceptado para publicación el 3 de diciembre de 2014) 